University of Nebraska - Lincoln

DigitalCommons@University of Nebraska - Lincoln

September 2001

\title{
The Blazhko Effect of the RR Lyrae Star DR Andromedae
}

Kevin M. Lee

University of Nebraska-Lincoln, klee6@unl.edu

Edward G. Schmidt

University of Nebraska-Lincoln, eschmidt1@unl.edu

Follow this and additional works at: https://digitalcommons.unl.edu/physicsschmidt

Part of the Physics Commons

Lee, Kevin M. and Schmidt, Edward G., "The Blazhko Effect of the RR Lyrae Star DR Andromedae" (2001). Edward Schmidt Publications. 5.

https://digitalcommons.unl.edu/physicsschmidt/5

This Article is brought to you for free and open access by the Research Papers in Physics and Astronomy at DigitalCommons@University of Nebraska - Lincoln. It has been accepted for inclusion in Edward Schmidt Publications by an authorized administrator of DigitalCommons@University of Nebraska - Lincoln. 
Publications of the Astronomical Society of the Pacific, 113:1140-1144, 2001 September

(C) 2001. The Astronomical Society of the Pacific. All rights reserved. Printed in U.S.A.

\title{
The Blazhko Effect of the RR Lyrae Star DR Andromedae
}

\author{
Kevin M. Lee and Edward G. Schmidt \\ Behlen Observatory, Department of Physics and Astronomy, University of Nebraska, Lincoln, NE 68588-0111; klee6@unl.edu, eschmidt1@unl.edu \\ Received 2000 June 18; accepted 2001 June 4
}

\begin{abstract}
We have obtained 551 new $V$ and $R$ observations of the RR Lyrae star DR Andromedae. Our data set is consistent with a primary period of 0.563118 days; however, the light curve of DR And cannot be well described by a single period. We conclude that DR And demonstrates the Blazhko effect with a Blazhko period of approximately 57.5 days.
\end{abstract}

\section{INTRODUCTION}

We have undertaken a survey of 107 Bailey type $a b$ RR Lyrae stars in an effort to determine the frequency and characteristics of multiperiodic behavior. We observe the stars near the phase of maximum light and identify multiperiodicity in those stars for which the magnitude or phase of maximum does not repeat well from one cycle to another. At this time, observations have been finished for about two-thirds of the survey sample and at least 15 new multiperiodic stars have been identified. Complete light curves are then obtained for the multiperiodic stars as time allows, and these will be published as they are completed rather than awaiting the conclusion of the survey. This is the third in a series of such papers, the first two being on V442 Her (Schmidt \& Lee 2000) and FM Per (Lee \& Schmidt 2001).

Most RR Lyrae stars repeat their light curves with remarkable regularity. However, roughly $30 \%$ of known RR Lyrae stars display variable light curves because of multiperiodicity; see the review by Szeidl (1988). The Blazhko effect (Blazhko 1907 ) is the cyclic modulation of either the amplitude alone or the amplitude and phase of maximum light over tens to hundreds of cycles. Stars that demonstrate variations of the amplitude alone often have very little light-curve variation at phases far from maximum. Smith (1995, Table 5.2) lists 46 stars known to exhibit the Blazhko effect. The Blazhko periods in this table range from 10.9 to 533 days but are typically between 20 and 200 days. There is no commonly accepted theory to explain the cause of the Blazhko effect. Hopefully, as the number of known RR Lyrae stars exhibiting the Blazhko effect increases, a correlation may be identified between the Blazhko effect and some other stellar parameter that could shed light on the physical mechanism causing the effect.

RR Lyrae stars may also have irregular light curves because of double-mode pulsation. These stars are known as RR $d$ stars (in the terminology of Nemec 1985). The mechanism for this variation (stars pulsating in the fundamental mode and the first overtone mode simultaneously) is well understood. Typically, the period ratio for these two processes, $P_{1} / P_{0}$, is around 0.743-0.748 (Cox 1987). Thus, light-curve variations due to double-mode pulsation manifest themselves on a shorter timescale than those due to the Blazhko effect, and it is easy to discriminate between the two. One would also expect lightcurve scatter from double-mode pulsation to be found not just near maximum light but at all phases. However, at present only three RR $d$ field stars have been identified (Jerzykiewicz \& Wenzel 1977; Clement, Kinman, \& Suntzeff 1991). An overview of these two phenomena and other less common types of multiperiodic behavior may be found in Kovacs (2001).

The star DR Andromedae (GSC 22860352) is located at $\alpha_{\mathrm{J} 2000}=1^{\mathrm{h}} 5^{\mathrm{m}} 10^{\mathrm{s}} .1$ and $\delta_{\mathrm{J} 2000}=34^{\circ} 13^{\prime} 10^{\prime \prime}$. Very little information exists on DR And in the literature. The General Catalogue of Variable Stars (GCVS; Kholopov 1985, 1987) lists a primary period of 0.563118 days and adds in the comments section that the period varies and the magnitude of maximum varies by 0.3 mag. It also lists values of $69.486,69.719$, and 2000 days and $30 \mathrm{yr}$ as being possible secondary periods. The only recently published observations were made by one of the authors (Schmidt, Chab, \& Reiswig 1995). Although Schmidt made only 15 observations of the star, he confirmed the star as a type RRab and found his data to be consistent with the GCVS period. He made no mention of any evidence for multiperiodicity.

\section{OBSERVATIONS}

We observed DR And 92 times between 1996 August 8 (JD 2,450,303) and 1997 February 19 (JD 2,450,498), an interval of 195 days. During a second observing run we obtained 459 points between 1997 June 17 (JD 2,450,616) and 1998 January 13 (JD $2,450,844$ ), an interval of 228 days. The observing system consisted of a Photometrics Ltd. TI4849, $390 \times 584$ pixel, cryogenically cooled CCD detector mounted at the Cassegrain focus of the Behlen Observatory $76 \mathrm{~cm}(\mathrm{f} / 13.5)$ telescope. The field of view is $2.9 \times 4 ! 3$, with a scale of 0.44 pixel $^{-1}$. Observations were made through $V$ and $R$ filters and transformed to the system 
TABLE 1

Coordinates and Magnitudes of the Comparison Stars

\begin{tabular}{cccccc}
\hline \hline \multicolumn{1}{c}{ Star } & GSC Number & $\alpha_{\mathrm{J} 2000}$ & $\delta_{\mathrm{J} 2000}$ & $V$ & $R$ \\
\hline $\mathrm{C} 1 \ldots \ldots$ & 22860613 & 1522.8 & 341259 & 13.366 & 12.818 \\
$\mathrm{C} 2 \ldots \ldots$ & 22860802 & 1517.5 & 341107 & 13.445 & 12.961 \\
\hline \hline
\end{tabular}

NOTE. - Units of right ascension are hours, minutes, and seconds, and units of declination are degrees, arcminutes, and arcseconds.

of Cousins (1976). Because of the large number of points involved, the original photometric data will not be listed here. It is available in the IAU Archives of Unpublished Observations of Variable Stars in archive 327E. See Breger, Jaschek, \& Dubois (1990) for a description of the archives and how to access them.

The Behlen Observatory CCD photometry system and observational techniques were the same as described earlier (Schmidt 1991 and references therein). However, it should be emphasized that differential magnitudes were calculated for DR And with other stars on the same CCD frames serving as comparison stars. Thus, accurate differential magnitudes were obtained even under inferior photometric conditions. On photometric nights, standard stars (from Landolt 1983) were observed, and color terms for the transformation to the standard system were derived. Average values of these color terms over intervals of several months were then used to correct all nights within the interval. Table 1 lists the coordinates and magnitudes on the standard system of the two comparison stars. The quality of the photometry may be judged by the rms scatter between the comparison stars, which was $17 \mathrm{mmag}$ in $V$ and 16 mmag in $R$.

\section{DATA ANALYSIS}

A period search was performed on the data using phase dispersion minimization (PDM; Stellingwerf 1978). In this method, the data are binned according to a series of trial periods and a statistic $\theta$ is calculated for each trial period; $\theta$ is defined as the weighted average of all individual bin variances divided by the variance of the entire data set. The data were grouped into phase bins of $\left(N_{b}, N_{c}\right)=(5,2)$ in Stellingwerf's nomenclature. The PDM periodogram obtained from the $V$ data set is shown in Figure 1, where a periodic signal yields a significant minimum in $\theta$. The most significant minimum corresponds to a period of 0.563175 days. The $R$ data set yielded an identical result.

However, the light curve obtained from the GCVS period of 0.563118 days was far superior to that produced from the period derived from our data using PDM. Thus, we adopted the GCVS period, and the resulting light curve formed from our $V$ data is shown in Figure 2. Visual inspection of this light curve is sufficient to convince one that DR And is multiperiodic and that the secondary periodicity has a fairly large amplitude. The phase of maximum light occurs at slightly larger phases when the magnitude of maximum light is larger. The mean intensity magnitudes of the light curves were determined to be $\langle V\rangle=12.460$ and $\langle R\rangle=12.227$.

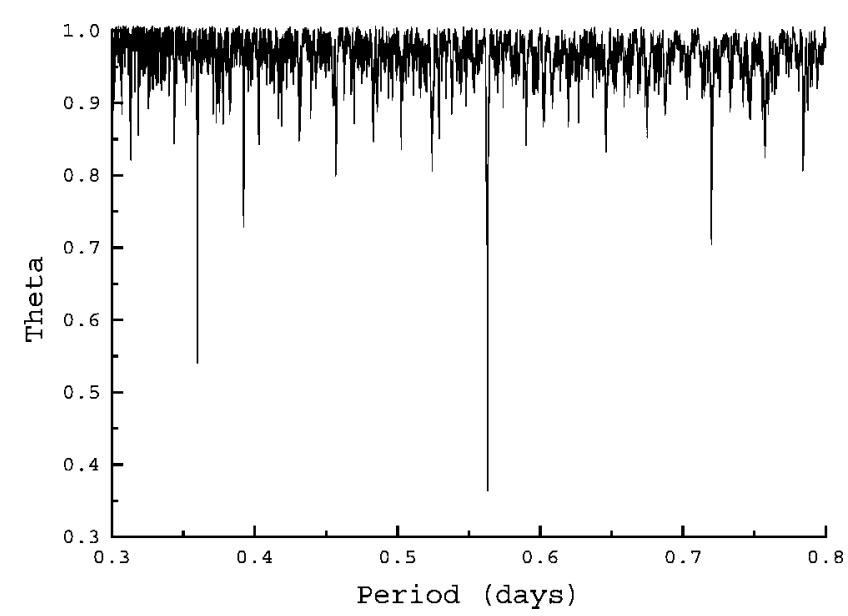

FIG. 1.-Power spectrum obtained from PDM analysis of the DR And $V$ data set.

We next investigated the variation in the magnitude of maximum light to determine the secondary periodicity. Table 2 lists the Julian Dates and magnitudes of DR And at maximum light obtained from the $V$ photometric data. Two different types of maximum light are identified: those for which the observations are sufficient in number and spacing so that the maximum is well defined (WD) and those for which the maximum may only be estimated (E). If not well defined, the magnitude of maximum light was estimated on a given night if there was a data point within 0.02 phase units of the phase of maximum light. The magnitude of the point within 0.02 phase units was adopted as the maximum value.

An examination of the values in Table 2 suggests that the Blazhko effect is present. There is one pair of maxima on 10,739 and 10,740 that were observed on successive nights. The magnitudes of maximum light are very similar, suggesting that the

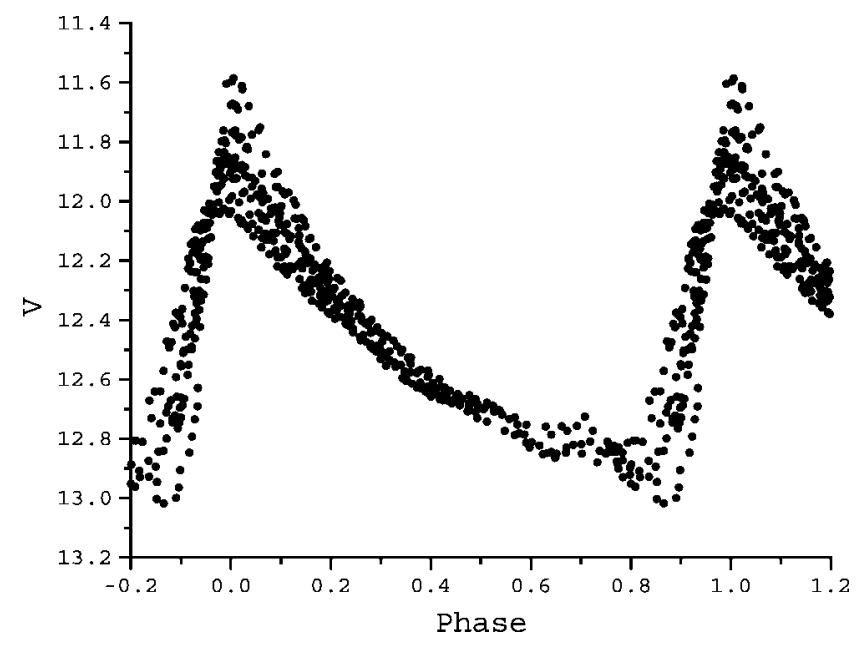

FIG. 2.-Light curve formed from the DR And $V$ data set where the phases have been determined by $\phi=(\mathrm{JD}-2,450,413.571) / 0.563118$. 
TABLE 2

Magnitudes of DR AND at Maximum Light

\begin{tabular}{|c|c|c|}
\hline $\begin{array}{l}\text { Heliocentric Julian Date } \\
\qquad(+2,440,000)\end{array}$ & $\begin{array}{c}V \text { Magnitude } \\
\text { (of Maximum Light) }\end{array}$ & Definition of Maximum \\
\hline $10405.678 \ldots$ & 11.672 & $\mathrm{E}$ \\
\hline $10413.573 \ldots \ldots$ & 11.672 & WD \\
\hline $10454.680 \ldots \ldots \ldots \ldots$ & 11.768 & WD \\
\hline $10480.577 \ldots \ldots$ & 11.850 & E \\
\hline $10498.594 \ldots \ldots \ldots \ldots$ & 12.025 & WD \\
\hline $10665.825 \quad \ldots \ldots \ldots \ldots \ldots$ & 12.026 & WD \\
\hline $10678.799 \ldots$ & 11.904 & WD \\
\hline $10692.877 \ldots$ & 11.597 & $\mathrm{E}$ \\
\hline $10709.776 \ldots \ldots$ & 11.853 & WD \\
\hline $10717.652 \ldots \ldots \ldots \ldots$ & 11.996 & WD \\
\hline $10726.655 \ldots \ldots \ldots \ldots$ & 12.031 & WD \\
\hline $10731.734 \ldots \ldots \ldots \ldots$ & 11.983 & WD \\
\hline $10739.616 \ldots \ldots \ldots \ldots$ & 11.875 & WD \\
\hline $10740.739 \ldots \ldots \ldots \ldots$ & 11.837 & $\mathrm{E}$ \\
\hline $10770.585 \quad \ldots \ldots \ldots \ldots \ldots$ & 11.870 & WD \\
\hline $10797.613 \ldots \ldots \ldots \ldots$ & 11.875 & WD \\
\hline $10814.514 \ldots \ldots \ldots \ldots$ & 11.586 & E \\
\hline
\end{tabular}

light curve does not change appreciably from one day to the next. This suggests that DR And exhibits the Blazhko effect rather than double-mode pulsation. The light curve in Figure 2 also suggests that the Blazhko effect is present since the lightcurve scatter is very pronounced near maxima and is much smaller at other phases. This phenomenon has been observed in many Blazhko stars such as RV UMa (Kanyo 1976). However, this behavior is not seen in RRd stars, where the secondary pulsation introduces scatter at all phases of the primary light cycle. From the data in Table 2, one can calculate that the amplitude of the Blazhko effect is at least 0.44 mag in $V$ and 0.40 mag in $R$.

Figure 3 graphs the data from Table 2 (the magnitudes of maximum light vs. Julian Date). One can see from the variation in the magnitudes of maximum light that the Blazhko period must be on the order of tens of days. We define a "Blazhko curve" as a graph whose abscissa is the phase of maximum light determined using the Blazhko period and the ordinate is the magnitude of maximum light. Thus, the Blazhko curve shows the magnitude at maximum light in the primary pulsation cycle plotted as a function of phase in the Blazhko cycle. To identify the Blazhko period, Blazhko curves were formed for a large number of trial Blazhko periods. The best Blazhko curves were formed using Blazhko periods in the range $57.5 \pm 0.2$ days. Figure 4 shows the Blazhko curve formed with phases determined by $\phi($ Blazhko $)=(\mathrm{JD}-2,450,665.825) / 57.5$.

We looked for supporting evidence of this Blazhko period using formal period search techniques. A mean light curve was formed using the LOWESS least-squares method (Cleveland 1981) and is shown superimposed over the primary $V$ light curve in Figure 5. The data were then prewhitened; the difference was calculated between the magnitude of each data

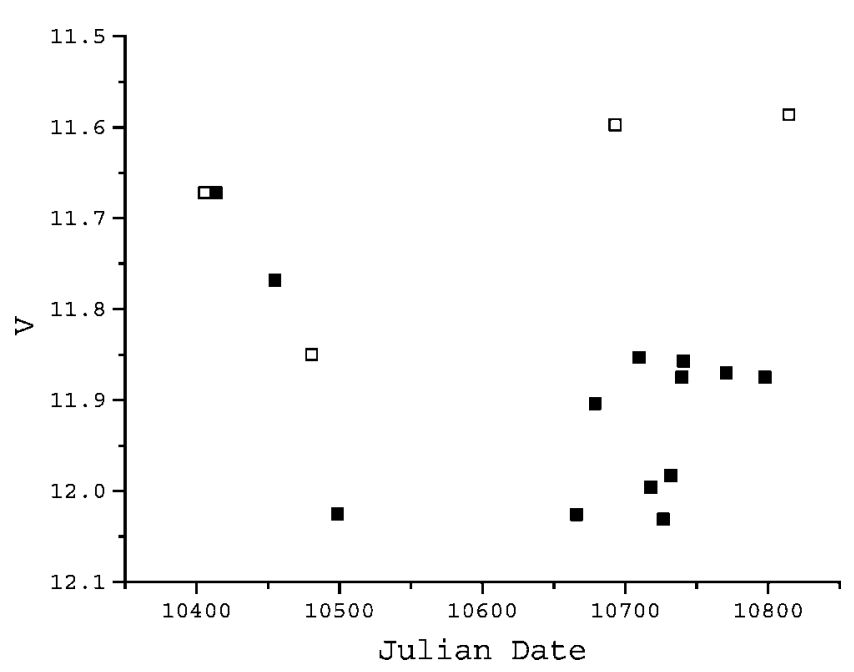

Fig. 3. $-V$ magnitudes of maximum light vs. Julian Date. The solid squares represent well-defined maxima, and the open squares represent estimated maxima.

point and the value of the mean curve at the same phase. The residuals versus phase are shown in Figure 6. One can see that very large residuals are obtained from data points on the ascending branch of the light curve.

The residuals were searched for periodicity using the PDM routine, and the periodogram for the period range below 1 day is shown in Figure 7. A minimum in $\theta$ is now observed at a period of 0.557656 days. Note that this is exactly the period one obtains from the beating of the Blazhko period against the

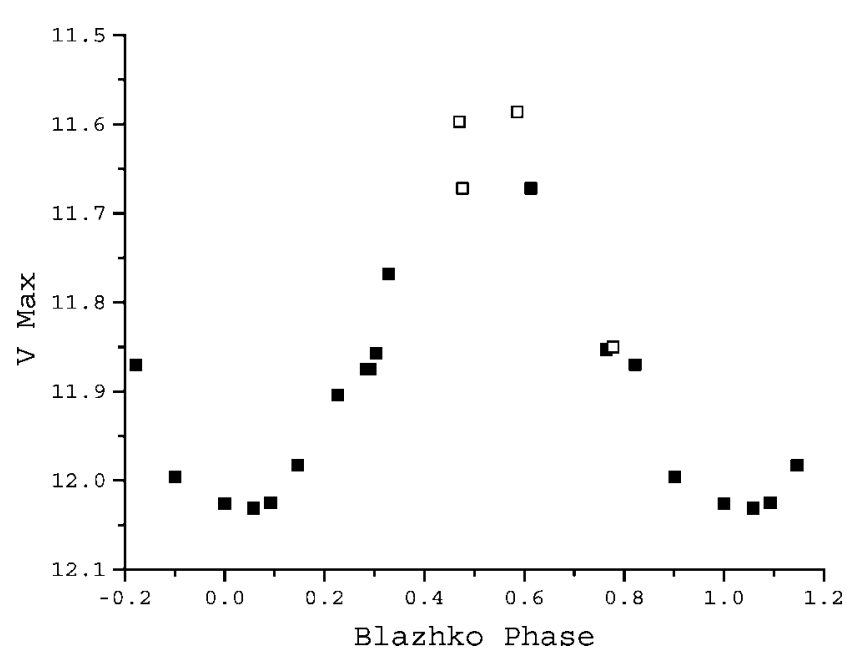

FIG. 4.-Blazhko curve formed from the $V$ magnitudes of maximum light. The Blazhko phases were determined from $\phi=(\mathrm{JD}-2,450,665.825) / 57.5$. The solid squares represent well-defined maxima, and the open squares represent estimated maxima. 


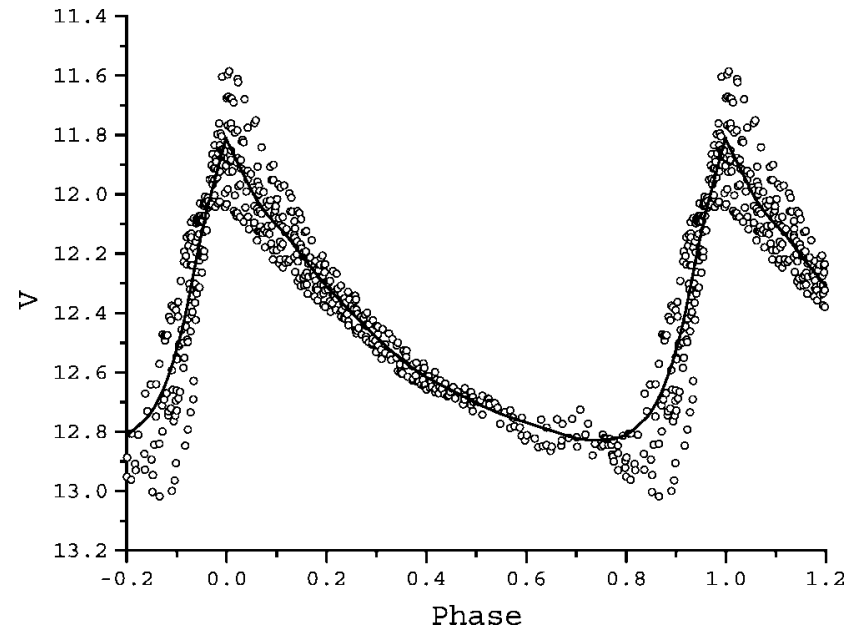

FIG. 5. $-V$ mean light curve superposed over the $V$ data set.

primary period:

$$
\begin{aligned}
P & =\left(\frac{1}{P_{0}}+\frac{1}{P_{B}}\right)^{-1}=\left(\frac{1}{0.563118 \text { days }}+\frac{1}{57.5 \text { days }}\right)^{-1} \\
& =0.557656 \text { days. }
\end{aligned}
$$

A period search performed on the light-curve maxima listed in Table 2 also supported this value of Blazhko period.

It is often problematic to determine Blazhko periods from power spectra for a number of reasons. The Blazhko effect is well known for being irregular (see Smith 1995, p. 107, for a discussion) and may not repeat itself precisely from one cycle to the next. The prewhitening residuals formed for data points on the ascending branch are by far the largest residuals. Thus, the particular sampling of these phases of the primary light curve inordinately governs the results of the secondary period search. This problem is exacerbated by the fact that the primary period used in the analysis may not be particularly accurate. The procedure also requires observations to be made over a very long baseline. Our two-season observing window spans 541 days. This encompasses 960 cycles of the primary period but only nine Blazhko cycles. Similar analysis procedures were applied to the star FM Per (Lee \& Schmidt 2001). This star has a Blazhko period of approximately 122 days, and no evidence of it could be found in power spectra analysis. Thus, secondary period search evidence is unlikely to convincingly determine Blazhko periods by itself except in cases of small Blazhko periods observed over very long baselines. The noisiness displayed in Figure 7 is typical of what one obtains when applying these procedures to Blazhko effect stars. Thus, although the secondary period search evidence presented above is far from conclusive by itself, it does support the value of the Blazhko period we obtained from forming the Blazhko curve.

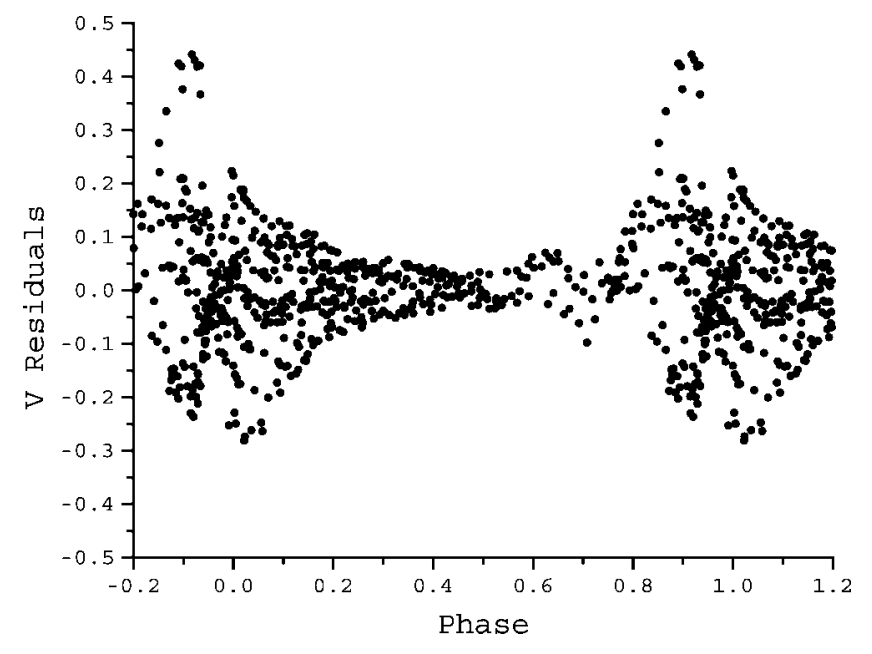

FIG. 6. $-V$ residuals formed by subtracting the $V$ mean light curve from the $V$ data at each phase.

\section{CONCLUSIONS}

It is clear that DR And is a multiperiodic RR Lyrae star. The variation in the magnitude of maximum light is caused by the Blazhko effect, and it is consistent with a Blazhko period of approximately 57.5 days.

The instrumentation used for the observations described in this paper was funded by the National Science Foundation (grant AST 85-04072). Support for publication expenses was provided by the American Astronomical Society through its small grants program. This research made use of the SIMBAD database operated at CDS, Strasbourg, France.

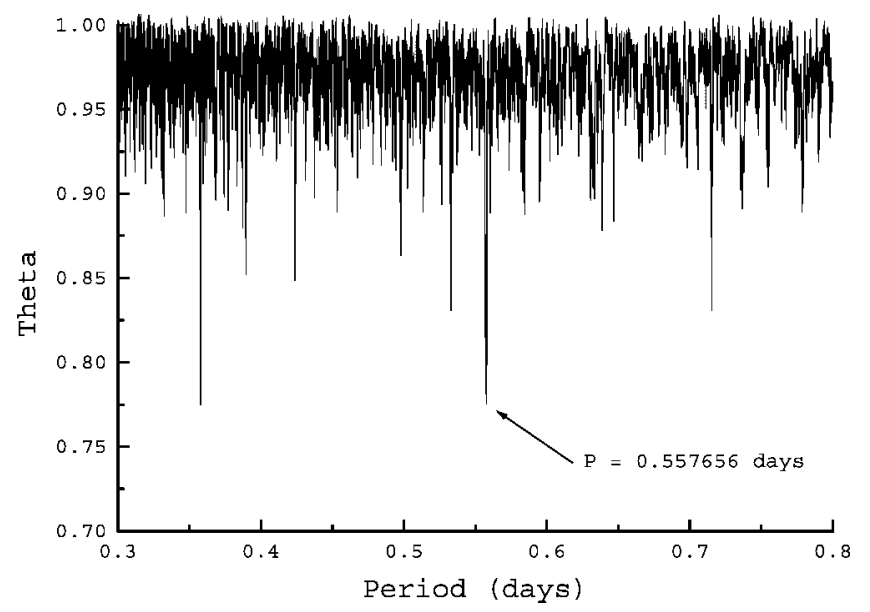

FIG. 7.-Power spectrum obtained from running PDM on the prewhitened residuals of the $V$ data set. 


\section{REFERENCES}

Blazhko, S. 1907, Astron. Nachr., 175, 325

Breger, M., Jaschek, C., \& Dubois, P. 1990, Inf. Bull. Variable Stars, 3422, 1

Clement, C. M., Kinman, T. D., \& Suntzeff, N. B. 1991, ApJ, 372, 273

Cleveland, W. S. 1981, Am. Stat., 35, 54

Cousins, A. W. J. 1976, MmRAS, 81, 25

Cox, A. N. 1987, in IAU Colloq. 95, Second Conf. on Faint Blue Stars, ed. A. G. D. Philip, D. S. Hayes, \& J. W. Liebert (Schenectady: Davis), 161

Jerzykiewicz, M., \& Wenzel, W. 1977, Acta Astron., 27, 35

Kanyo, S. 1976, Mitt. Sternw. Ungarischen Akad. Wiss. Budapest, 69

Kholopov, P. N. 1985, General Catalogue of Variable Stars (4th ed.; Moscow: Nauka)
1987, General Catalogue of Variable Stars, Vol. 3 (4th ed.; Moscow: Nauka)

Kovacs, G. 2001, in Stellar Pulsation-Nonlinear Studies, ed. M. Takeuti \& D. Sasselov (Dordrecht: Kluwer)

Landolt, A. U. 1983, AJ, 88, 439

Lee, K. M., \& Schmidt, E. G. 2001, PASP, 113, 834

Nemec, J. M. 1985, AJ, 90, 204

Schmidt, E. G. 1991, AJ, 102, 1766

Schmidt, E. G., Chab, J. R., \& Reiswig, D. E. 1995, AJ, 109, 1239

Schmidt, E. G., \& Lee, K. M. 2000, PASP, 112, 1262

Smith, H. A. 1995, RR Lyrae Stars (Cambridge: Cambridge Univ. Press)

Stellingwerf, R. F. 1978, ApJ, 224, 953

Szeidl, B. 1988, in Multimode Stellar Pulsations, ed. G. Kovacs, L. Szabodos, \& B. Szeidl (Budapest: Konkoly Obs.), 45 